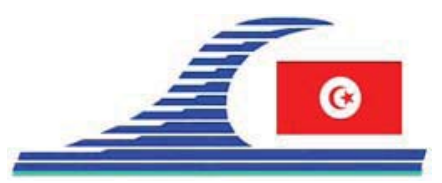

Conférence Méditerranéenne Côtière et Maritime EDITION 1, HAMMAMET, TUNISIE (2009)

Coastal and Maritime Mediterranean Conference

Disponible en ligne - http://www.paralia.fr-Available online

\title{
Étude des digues partiellement immergées au cas des faibles profondeurs avec passage de courant
}

\author{
Michel BÉLORGEY ${ }^{1}$, Christophe COLMARD ${ }^{2}$, Joël KOUAM-KOM ${ }^{3}$
}

1. Association POSEIDOM, Parc du Chapitre, 76420 Bihorel, France. belorgey@poseidom.fr

2. SAIPEM S.A., Energies, 1/7 avenue San Fernando, Montigny-le-Bretonneux, 78884 Saint Quentin en Yvelines, France.

christophe.colmard@saipem-sa.com

3. Université de Caen, Caen, France.

\section{Résumé :}

Les ouvrages classiques de protection des ports ou des côtes sont des digues ou des épis bâtis à partir du fond. S'ils protègent de la houle, ils constituent aussi un obstacle au transfert, par le courant, des sédiments ou matières organiques indispensables à la nutrition des espèces aquatiques.

Dans le cadre de l'extension du port de Monaco, où les profondeurs sont importantes $(60 \mathrm{~m})$, la Société Bouygues Offshore (SAIPEM SA) a lancé des recherches visant à mieux comprendre et à améliorer la solution "Mur d'eau fixe » de BOUCHET et MANZONE (COLMARD, 1997). Les travaux de COLMARD et BÉLORGEY (1996), ont permis de mettre au point le fameux caisson BYBOP breveté en 1997, (digue partiellement immergée montée sur pieux). Ce système limite les phénomènes de résonance observés jusqu'alors dans les sites fortement réfléchissants, mais surtout permet d'obtenir de meilleurs rendements en termes de réflexion et de transmission.

Nous avons analysé les performances du caisson BYBOP dans le cas des faibles profondeurs avec passage de courant et plus particulièrement dans le cas du port d'Anzali (Iran) situé à l'embouchure d'un estuaire qui relie un lagon à la mer. L'objectif étant de protéger le port sans perturber le transfert hydraulique entre le lagon et la mer.

Les résultats que nous présentons ici montrent que le système BYBOP est tout à fait adapté à ce problème. Il protège de la houle sans perturber le courant et les transferts entre le lagon et la mer.

\section{Mots-clés :}

Génie côtier - GIZC - Hydraulique maritime - Écosystèmes Estuariens - Ecosystèmes côtiers - BYBOP

\section{Introduction}

Le port d'Anzali (Iran) est situé dans la partie sud-ouest de la Mer Caspienne (voir figure 1a). La richesse de cette région n'est pas uniquement dans le pétrole, mais aussi DOI: $10.5150 / \mathrm{cmcm} .2009 .002$ 
dans les ressources de la pêche en particulier en ce qui concerne l'esturgeon (le caviar est l'une des grandes richesses de l'Iran). Ce port est situé à l'embouchure d'un estuaire qui relie la Mer Caspienne à une lagune très vaste de $450 \mathrm{~km}^{2}$ (plus de $40 \mathrm{~km}$ de long et $20 \mathrm{~km}$ de large). La protection des espèces aquatiques de ce secteur exige de préserver les échanges et le transfert des masses d'eau entre la lagune et la mer.

La configuration du port d'Anzali prend en compte ce besoin, mais par contre il subit de plein fouet les vagues du nord et du nord-ouest qui dans ce secteur sont les plus importantes.

Un projet de digue détachée type BYBOP a été proposé (figure 1b), permettant ainsi de protéger le port d'Anzali sans perturber les échanges et transferts entre le lagon et la mer.

Les études réalisées ont montré que le système BYBOP répondait très bien aux conditions de protection et de transfert des masses d'eau.

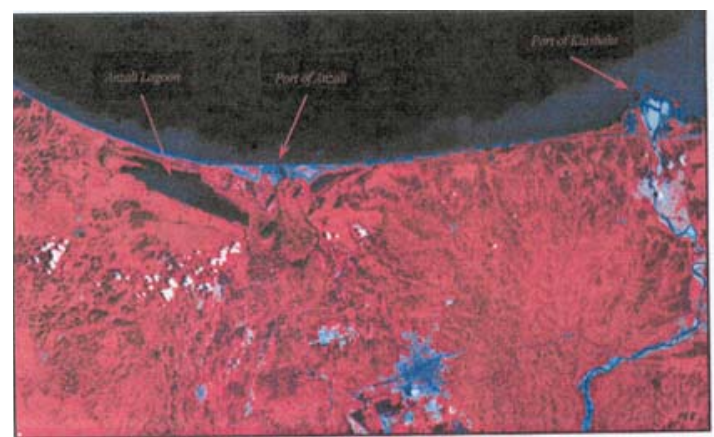

(1a)

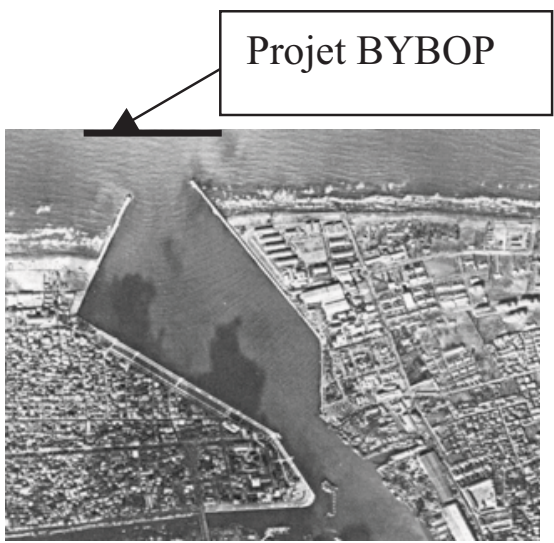

(1b)

Figure 1. Position géographique du port d'Anzali et position du projet BYBOP.

\section{Conditions expérimentales}

Les expériences ont été menées en canal à houle avec courant (longueur $16.5 \mathrm{~m}$, profondeur $0.6 \mathrm{~m}$, largeur $0.5 \mathrm{~m}$ ). Le générateur de houle était de type piston. Le courant était produit par une pompe hélice et la veine fluide fonctionnait en circuit fermé grâce à une conduite de retour. La pompe hélice fonctionnant dans un sens ou dans l'autre de manière à avoir deux sens du courant :

- L'un dans le sens de la propagation de la houle [C+].

- L'autre dans le sens inverse à celui de la propagation de la houle [C-].

Pour les différentes conditions, les paramètres de la houle ont été mesurés à l'aide de sondes de surface libre classiques et analysés en utilisant la méthode de MANSARD et FUNKE (1980). Et les vitesses au sein de la masse fluide ont été mesurées par un vélocimètre laser à deux composantes DANTEC. 
Tableau 1. Conditions de similitude.

\begin{tabular}{|c|c|c|c|c|c|c|}
\hline & $\begin{array}{l}\text { Période } \\
T(s)\end{array}$ & $\begin{array}{l}\text { Houle } \\
\text { incidente } \\
\text { Hi (m) }\end{array}$ & $\begin{array}{l}\text { Tirant } \\
\text { d'eau } \\
\text { I (m) }\end{array}$ & $\begin{array}{l}\text { Prof. } \\
h(m)\end{array}$ & $\begin{array}{l}\text { Largeur } \\
\text { caisson } \\
W(m)\end{array}$ & $\begin{array}{l}\text { Vitesse } \\
\text { courant } \\
U(\mathrm{~m} / \mathrm{s})\end{array}$ \\
\hline $\begin{array}{l}\text { Prototype } \\
\text { ANZALI }\end{array}$ & $5-10$ & $1-2$ & $3-5$ & 8 & 20 & 0.5 \\
\hline $\begin{array}{l}\text { Modèle } \\
\text { physique }\end{array}$ & $0.88-1.77$ & $0.031-0.063$ & $0.094-0.156$ & 0.25 & 0.625 & 0.09 \\
\hline
\end{tabular}

Le positionnement de la maquette dans le canal est donné sur la figure 2.

Les études ont été réalisées pour diverses conditions de houle et de courant :

- Houle seule $(\mathrm{H})$.

- Houle et courant dans le sens de propagation de la houle $(\mathrm{H}+\mathrm{C})$.

- Houle et courant dans le sens opposé à la propagation de la houle (H-C).

Elles sont présentées dans le tableau 2.

Tableau 2. Conditions expérimentales.

\begin{tabular}{rrrrrr}
\hline & $\boldsymbol{T}(\mathbf{s})$ & $\boldsymbol{H i}(\boldsymbol{m})$ & $\boldsymbol{H t}(\boldsymbol{m})$ & $\boldsymbol{C r}(\%)$ & $\boldsymbol{C t}(\%)$ \\
\hline \multirow{4}{*}{$\boldsymbol{H}$} & 1.33 & 0.049 & 0.0071 & 0.5327 & 0.1566 \\
& 1.52 & 0.051 & 0.0092 & 0.5185 & 0.2047 \\
& 1.68 & 0.060 & 0.0160 & 0.5557 & 0.2510 \\
1.83 & 0.024 & 0.0070 & 0.6270 & 0.3150 \\
\hline & 1.33 & 0.042 & 0.0036 & 0.4923 & 0.0891 \\
& 1.52 & 0.047 & 0.0100 & 0.4646 & 0.2027 \\
$\boldsymbol{H}+\boldsymbol{C}$ & 1.68 & 0.052 & 0.0155 & 0.3982 & 0.3011 \\
& 1.83 & 0.025 & 0.0070 & 0.4777 & 0.2680 \\
\hline & 1.33 & 0.036 & 0.0043 & 0.6037 & 0.1290 \\
$\boldsymbol{H}-\boldsymbol{C} 1.68$ & 0.046 & 0.0162 & 0.6624 & 0.3202 \\
1.83 & 0.023 & 0.0069 & 0.7261 & 0.2976 \\
\hline
\end{tabular}

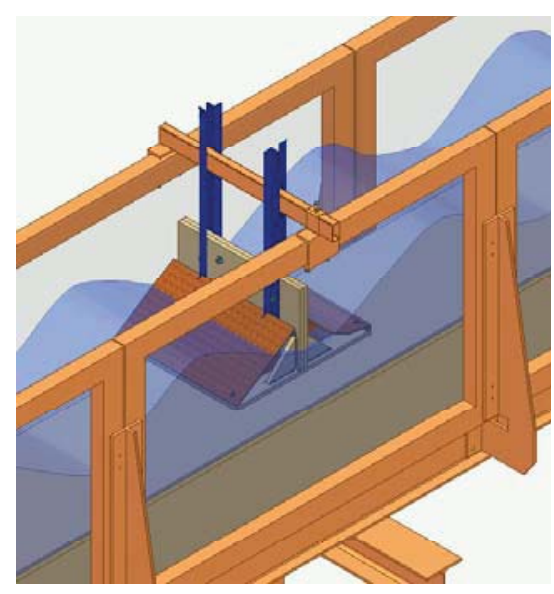

Figure 2. Maquette BYBOP dans canal à houle.

\section{Résultats expérimentaux}

Pour chacune des conditions, nous avons analysé pour chaque houle incidente Hi :

- La houle transmise à l'arrière du BYBOP (Ht) et le coefficient de transmission

- La houle réfléchie à l'avant du BYBOP (Hr)

- Le champ des vitesses dans 5 profils P1 et P2 à $1.5 \mathrm{~m}$ et $0.4 \mathrm{~m}$ avant la structure, $\mathrm{P} 3$ sous la structure, $\mathrm{P} 4$ et $\mathrm{P} 5$ à $-0.4 \mathrm{~m}$ et $-1.5 \mathrm{~m}$ à l'arrière de la structure 
La figure 3 montre l'évolution du coefficient de transmission $\mathrm{Ct}=\mathrm{Ht} / \mathrm{Hi}$ de la houle à l'arrière du caisson BYBOP. Et la figure 4 montre le profil des vitesses moyennes associées au courant (ici $\mathrm{C}+$ ) dans chaque profil.

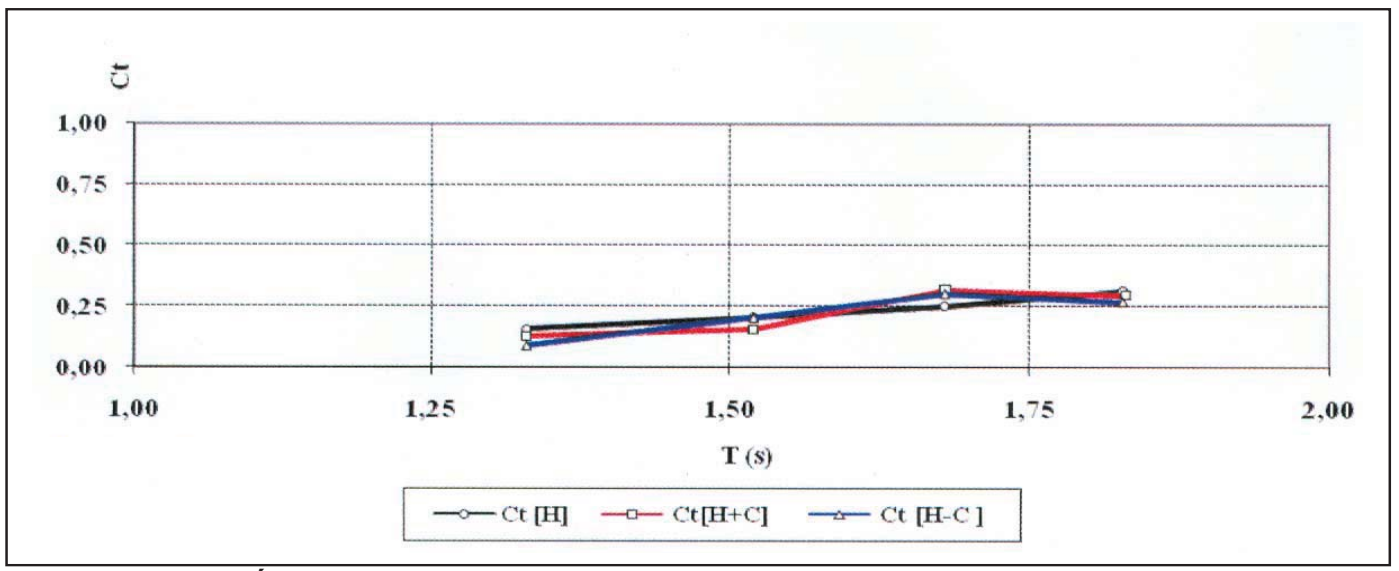

Figure 3. Évolution du coefficient Ct en fonction de la période de la houle.

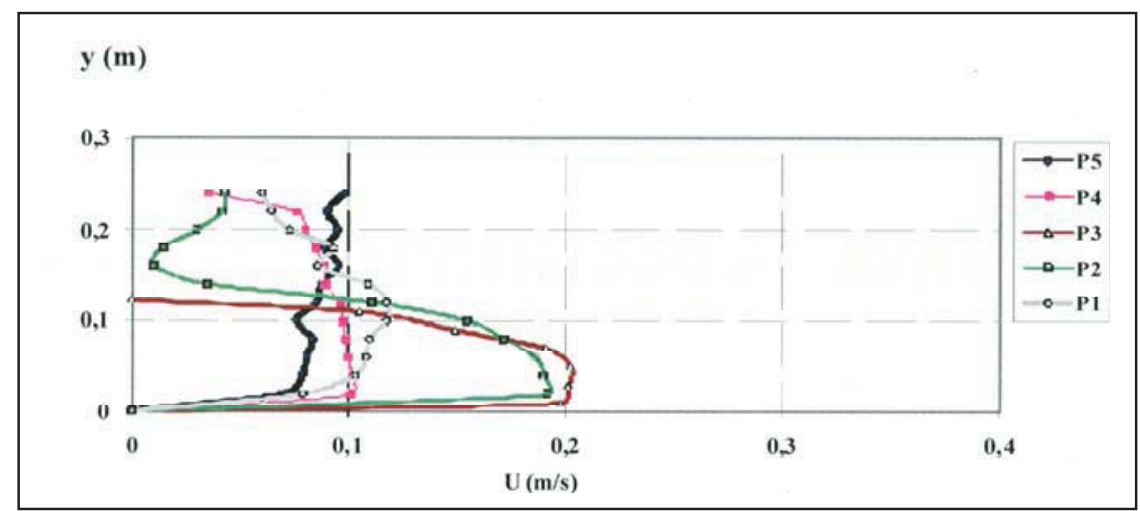

Figure 4. Champ des vitesses moyennes pour les différents profils.

\section{Conclusions}

Les résultats obtenus ainsi que les vidéos que nous avons réalisées montrent que le caisson BYBOP est un système de protection efficace des côtes qui ne perturbe pas les transferts des masses d'eau.

\section{Références bibliographiques}

COLMARD C., BELORGEY M. (1996). Resonance phenomenon developed by breakwater or breakwater fixed on piles. $7^{\text {th }}$ ISOPE (International Offshore and Polar Engineering Conference), Honolulu, USA.

COLMARD C. (1997). Etude du phénomène "Mur d'Eau Fixe". Thèse de Doctorat, Université du Havre.

MANSARD E.P.D., FUNKE, E.R. (1980). The measurement of incident and reflected spectra using a least squares method. Proc. of the 17th Coastal Engineering Conf., ASCE, pp 154-172. 